\title{
Focal low-dose rate brachytherapy for the treatment of prostate cancer
}

\author{
This article was published in the following Dove Press journal: \\ Cancer Management and Research \\ 12 September 2013 \\ Number of times this article has been viewed
}

\section{William Y Tong \\ Gilad Cohen \\ Yoshiya Yamada}

Department of Radiation Oncology, Memorial Sloan-Kettering Cancer

Center, New York, NY, USA
Correspondence: Yoshiya Yamada

Department of Radiation Oncology, Memorial Sloan-Kettering Cancer Center, I 275 York Avenue, Box 22, New York, 10065, NY, USA

Tel +I 2126392950

Fax + I 2126392417

Email yamadaj@mskcc.org
Abstract: Whole-gland low-dose rate (LDR) brachytherapy has been a well-established modality of treating low-risk prostate cancer. Treatment in a focal manner has the advantages of reduced toxicity to surrounding organs. Focal treatment using LDR brachytherapy has been relatively unexplored, but it may offer advantages over other modalities that have established experiences with a focal approach. This is particularly true as prostate cancer is being detected at an earlier and more localized stage with the advent of better detection methods and newer imaging modalities.

Keywords: prostate cancer, focal, low dose rate, brachytherapy

\section{Introduction}

Prostate cancer is the most common malignancy in men, with an estimated incidence in the United States of 241,740 cases and 28,170 deaths in 2012 (Surveillance, Epidemiology and End Results [SEER] stats). ${ }^{1}$ Low-dose rate (LDR) brachytherapy has been an effective form of definitive therapy for low-risk localized prostate cancer with excellent long-term outcomes. ${ }^{2-5}$ Traditionally, LDR brachytherapy has been delivered to the whole gland of the prostate. While the outcomes have proven successful, it is not without adverse effects that could include urinary, rectal, and sexual toxicities that may affect a patient's quality of life. ${ }^{6}$ Treating a portion of the gland or a focal region of well-identified disease within the prostate gland may offer an option of reducing the toxic effects of treatment while maintaining similar treatment outcomes when compared with whole-gland therapy. Advantages in quality of life could be exhibited in the form of reduced urinary incontinence, rectal symptoms, and improved erectile and prostatic gland function.

Approaches to partial-prostate gland treatment include hemigland therapy, the addition of a focal boost to whole-gland therapy, and finally treatment of a focal region alone. These distinctions are important, as the term focal therapy at times has been used interchangeably in the past to represent one of these partial approaches.

The experience with focal LDR brachytherapy in prostate cancer to date is limited. There are ongoing trials evaluating hemigland and focal approaches in selected patients, ${ }^{7,8}$ and there has been experience with delivering LDR brachytherapy to the peripheral zone alone. ${ }^{9}$ Much of the experience with a focal approach in itself has been seen with ablative techniques that include high-intensity focused ultrasound (HIFU) and cryotherapy. Other, less-studied partial approaches to deliver radiotherapy include hemigland high-dose rate (HDR) brachytherapy ${ }^{10}$ and a focal boost to a course of 
whole-gland radiation. In the latter experiences, the focal boost has been delivered with differential dose external beam radiation, differential HDR brachytherapy, and LDR brachytherapy. ${ }^{11-14}$

Selecting appropriate patients for focal therapy will be a vital component of exploring this therapeutic modality as an option. Prostate cancer is being detected at an earlier and more localized stage of disease with the widespread use of prostate-specific antigen (PSA) detection as well as improvement in biopsy and imaging techniques. ${ }^{15}$ Such advances offer improvements in selecting patients with focal low-disease burden while excluding those with multifocal disease to preferentially select patients for focal treatment. ${ }^{16,17}$

Here, we review various partial and focal therapy experiences to date, evaluate the advancements in disease detection and their role in the future of selecting patients for focal LDR brachytherapy, and assess aspects of LDR brachytherapy delivery in the context of focal treatment.

\section{Focal therapy experience}

Much of the experience with focal therapy to date has been with ablative techniques that include HIFU and cryotherapy. In properly selected groups, these techniques have shown good results in terms of outcomes and toxicities. For example, in one focal HIFU experience, a 2-year biochemical diseasefree survival rate of $83.3 \%$ was reported. ${ }^{18}$ It was observed that the focally treated group had decreased the indwelling catheter period, frequency of urinary stricture, and urinary tract infection in comparison to whole-gland treatment. In another series described as hemiprostate HIFU ablation, the 10 -year overall survival was reported at $83 \%$ and cancerspecific survival at $100 \%$. All patients maintained urinary continence. ${ }^{19}$ In the focal cryotherapy experience, one report indicated a biochemical disease-free survival rate of $88 \%$ over a median follow-up time of 28 months, with maintenance of continence and potency at $71 \%$. In another experience, the biochemical disease-free survival rate was $95 \%$ over a mean follow-up time of 3.6 years, with maintenance of continence and sexual potency rates of $85 \%{ }^{20-22}$ These experiences and other focal HIFU and cryotherapy experiences are summarized in Table 1.

The dosimetric feasibility to plan hemigland HDR brachytherapy has been evaluated. In this study, hemigland treatment plans were compared to whole-gland treatment plans. Hemigland dosimetric assessment revealed effective target coverage, and doses to the rectum, bladder, and urethra were reduced significantly. The degree of reduction in the dose to $2 \mathrm{cc}$ to these organs was from $64.1 \%-53.1 \%$ for the rectum, $67.5 \%-55.9 \%$ for the bladder, and $95.2 \%-69.3 \%$ to the urethra. ${ }^{10}$ The addition of a focal radiation boost by stereotactic radiation or HDR brachytherapy to a course of whole-gland external beam radiation has also been studied. ${ }^{11-13}$ In one experience, patients received a conventionally fractionated external radiation dose to 64-64.4 Gy followed by a hypofractionated course of 5-7 Gy in two fractions delivered stereotactically. ${ }^{11}$ In another experience, a hypofractionated course of external radiation was delivered stereotactically to a dose of 38 Gy in four daily fractions, followed by an integrated boost to 11 Gy per fraction to the dominant lesion if visible on magnetic resonance imaging (MRI). ${ }^{12}$ With HDR brachytherapy as a partial boost, one experience treated patients with external beam radiation doses of 64-64.4 Gy, followed by either a bilateral or unilateral HDR brachytherapy boost. Unilateral HDR boost doses ranged from $12-16$ Gy in two fractions. ${ }^{13}$ LDR brachytherapy to the whole gland with a concomitant brachytherapy boost to identified lesions has been evaluated as well. In these experiences, the feasibility of using magnetic-resonance spectroscopy imaging (MRSI) to identify tumor deposits and deliver additional dose to these regions with $\mathrm{I}^{125}$ seeds was demonstrated. Improved estimated tumor control probability was shown while maintaining acceptable dose constraints to the urethra. ${ }^{14}$ While the outcomes of focal boost added to whole-gland treatments cannot easily be compared to hemigland or focal experiences, acceptable and, in some cases, reduced toxicity were demonstrated and provided a basis for a movement toward a focal delivery of radiation.

LDR brachytherapy for prostate cancer has been used for a number of decades with the majority of recent experiences emerging from the 1980s with the introduction of transrectal ultrasound to plan and guide the delivery of treatment. LDR brachytherapy has traditionally been delivered to the whole gland with excellent long-term outcomes. Fifteen-year biochemical relapse-free survival has been reported at $85.9 \%$ for low-risk patients ${ }^{3}$ and 10-year disease-specific survival at $96 \%{ }^{4}$ Toxicities primarily involve urinary, rectal, and sexual function. Urinary symptoms exhibited as mild obstructive or irritative symptoms may be seen in $50 \%$ of patients in the immediate postimplant period, with $90 \%$ returning to their baseline urinary function by 12 months, and $<3 \%$ requiring surgical management to relieve obstructive urinary symptoms long term. ${ }^{23-26}$ Rectal irritation may be seen in up to $30 \%$ of patients within the first 2 years which resolves, while rectal bleeding may be seen in up to $7 \%$ of patients, and rectal fistulas in $<1 \%$ of patients. ${ }^{27,28}$ Erectile function is maintained in $50 \%-80 \%$ of patients with variations 


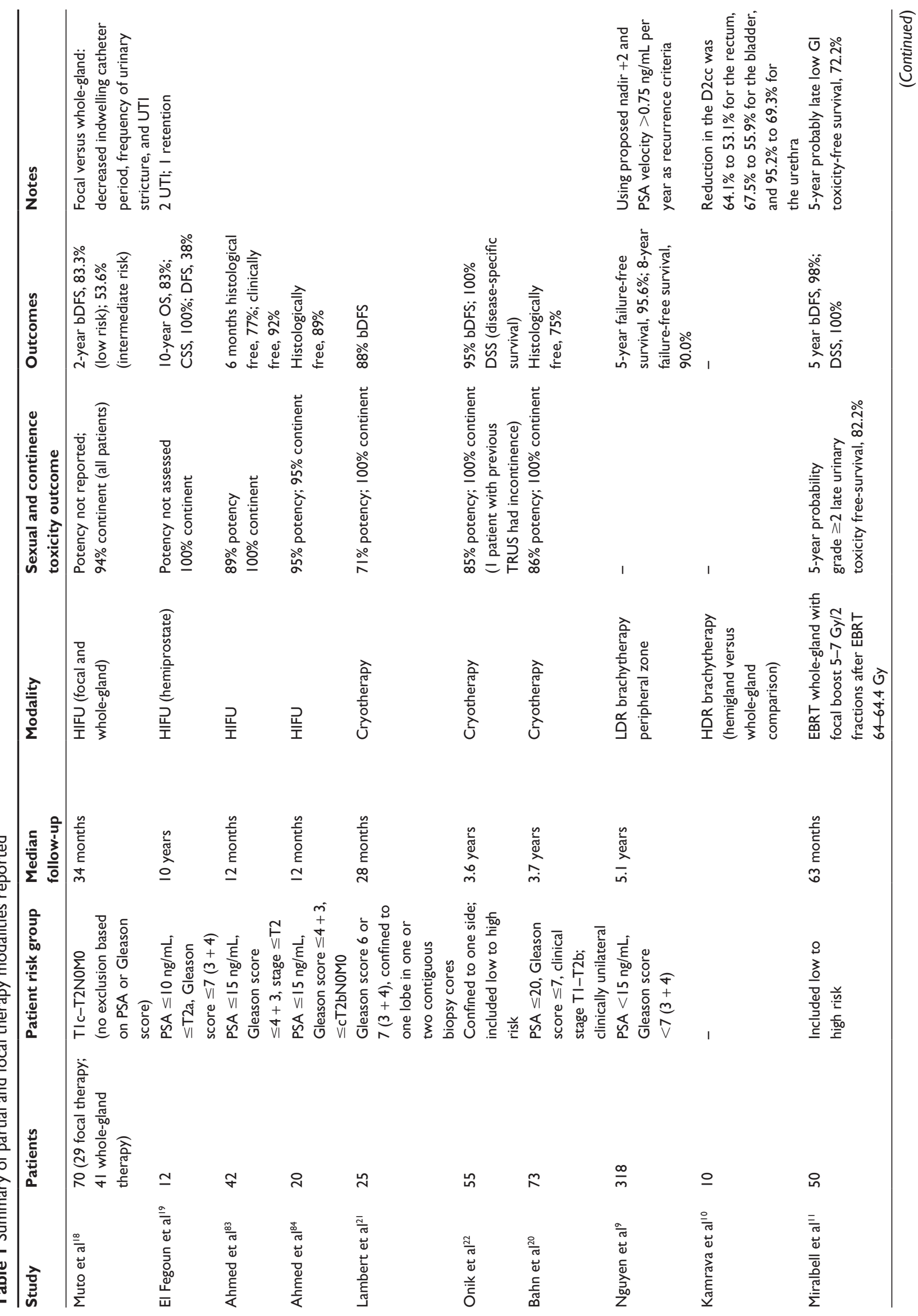




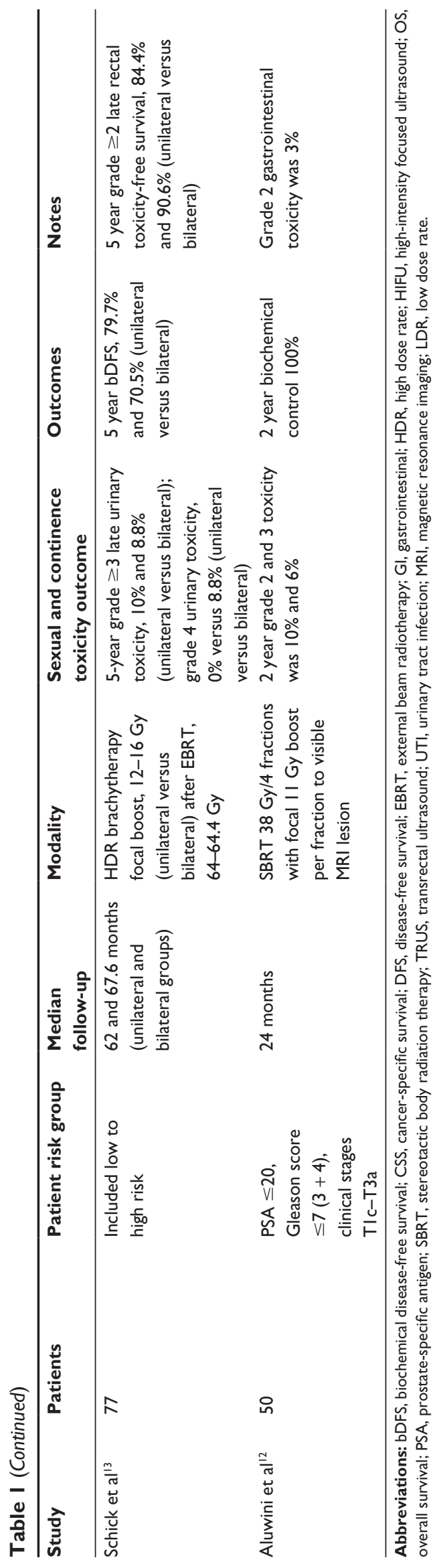

dependent on pretreatment erectile function, age at the time of treatment, and pharmacologic assistance. ${ }^{25,29}$ While the use of LDR brachytherapy for whole-gland treatment is very well established, there is very limited data with its use in focal-only treatment. There is one experience in which LDR brachytherapy was delivered to the peripheral zone alone. The PSA failure-free survival for low-risk patients at 5 and 8 years was $95.6 \%$ and $90.0 \%$, respectively. ${ }^{9}$ The successful experience and established dose response with whole-gland LDR brachytherapy, ${ }^{5,30}$ along with acceptable toxicities, may have reduced the motivation toward a focal approach. However, with the advent of better disease detection and the potential for further minimizing toxicity, greater experience with focal LDR brachytherapy should be considered. As such, its use in a focal manner is currently explored with protocols looking at hemigland ${ }^{8}$ and focal LDR brachytherapy. ${ }^{7}$

\section{Selection of appropriate patients}

Selecting appropriate patients for focal therapy would be of utmost importance, specifically patients with low-disease burden that is confined to a portion of the prostate gland. Advances in ultrasound and MRI techniques, as well as tissue sampling, have enhanced the ability to select these patients. Advances in ultrasound techniques include B mode ultrasound, color Doppler imaging, and contrast-enhanced ultrasound. These techniques can assist in localizing tumors based on vascular variance and tumor tissue differences. ${ }^{31-34}$ Ultrasound imaging, based on spectrum analysis of radiofrequency signals, has shown good biopsy correlation. ${ }^{35,36} \mathrm{In}$ addition, there is some evidence that elastography and histoscanning may add diagnostic value as well..$^{37,38}$

The use of MRI is gaining in popularity for the diagnosis and planning of prostate therapy. Localization of tumors within the prostate gland has improved with the use of multiparametric MRI, which adds functional sequences that include diffusion weighting, dynamic contrast enhancement, and MRI spectroscopy. It has been reported that dynamic contrast-enhanced imaging has a sensitivity of $86 \%-90 \%$ and a specificity of $88 \%-94 \%$ in identifying a tumor sized at $0.5 \mathrm{~mL} \cdot{ }^{39-41}$ As well, identification of tumors down to $0.2 \mathrm{~mL}$ in size has been reported, particularly in the intracapsular transition or peripheral zone. ${ }^{39-41}$ MRI spectroscopy has shown a sensitivity of $91.9 \%$ and specificity of $98.3 \%{ }^{42}$ When correlated with Gleason score, MRI has been shown to have good sensitivity and specificity in identifying tumors with more aggressive disease. ${ }^{43,44}$ One important consideration is that MRI results may be obscured by biopsy changes, and it is recommended that MRI evaluation should be performed 
either prior to biopsy or no earlier than 6 weeks following biopsy. ${ }^{45}$

With respect to tissue diagnosis, optimizing tissue sampling would also be imperative in identifying populations appropriate for focal therapy. One aspect to consider is the inhomogeneity in tissue sampling and interpretation standards. This includes the variability in the number of cores taken, biopsy length, and location sampled by the clinician as well as tissue processing and interpretation by the pathologist. ${ }^{46,47}$ This may lead to undergrading in as many as $30 \%$ of the cases and understaging in as many as $25 \%$ of the cases. ${ }^{48}$ As a result, guidelines for the standardization and optimization of tissue sampling would be an important component of candidacy for focal therapy.

The common standard for prostate biopsy involves six to twelve transrectal biopsy cores. It has been shown that this may not be adequate for identifying patients for focal therapy, as it may result in missing the disease in $31 \%$ of cases. ${ }^{49,50}$ MRI-guided biopsy could be considered, as it has been shown to have greater detection rate when compared to transrectal ultrasound-guided biopsy and has a detection rate of $59 \%$ after an initial negative biopsy. ${ }^{51} \mathrm{~A}$ better method of pathologically mapping the prostate is by transperineal threedimensional mapping biopsy with a $5 \mathrm{~mm}$ template. ${ }^{52}$ This has been shown to identify bilateral disease in $39 \%$ of cases that were found to be negative on transrectal ultrasoundguided biopsy. ${ }^{48}$

Review of biopsy findings suggests that the expected number of cases with unifocal disease may be in the range of $13 \%-35 \%{ }^{53,54}$ Of those with low-risk unifocal disease, only $1 \%$ displayed extracapsular extension. ${ }^{55}$ In cases where multifocal disease was observed, it was found that $97 \%$ of index lesions displayed the same Gleason score as the overall cancer. ${ }^{56}$

While advances in imaging techniques as well as tissue sampling will help identify patients appropriate for focal therapy, other factors may need to be considered. These additional factors can help select patients who are likely to have minimal toxicity from brachytherapy and may have lowrisk, low-volume disease that could be appropriate for focal therapy. A multidisciplinary international consensus group has proposed a number of factors to consider, which include life expectancy, PSA burden, the use of multiparametric MRI, the use of bilateral template-guided prostate-mapping biopsy, unilateral disease, Gleason score of 6-7, and tumor stage ( $\leq$ T2b) (Table 2). ${ }^{57}$ In comparison, in the Phase II hemigland brachytherapy protocol, the inclusion criteria include life expectancy, pathology, performance status, stage
Table 2 Multidisciplinary international consensus group proposal selection

Life expectancy $>10$ years

PSA $\leq 15 \mathrm{ng} / \mathrm{mL}$

Multiparametric (TI W/T2 W score, diffusion-weighting, dynamic

contrast enhancement \pm spectroscopy)

Magnetic resonance imaging prior to biopsy

Bilateral template-guided prostate mapping biopsy with $5 \mathrm{~mm}$ sampling frame

Unilateral disease; lesion size $\leq 0.5 \mathrm{~mL}$ (approximately equates to maximum cancer length of $10 \mathrm{~mm}$ ) with or without clinically insignificant disease on the contralateral side (cancer core length $\leq 3 \mathrm{~mm}$ )

Gleason score of index lesion 6-7 $(3+4)$

Tumor stage $\leq \mathrm{T} 2 \mathrm{~b}$

Prostate size $\leq 60 \mathrm{~mL}$

Abbreviation: PSA, prostate-specific antigen.

(T1c-T2a), PSA value, MRI evidence of unilateral disease, and prostate size (Table 3). ${ }^{8}$ As well, the exclusion criteria for this protocol include inability to tolerate anesthesia, extracapsular extension involvement, International Prostate Symptom Score (IPSS), ${ }^{58}$ ability to undergo MRI, and prior radiation (Table 4).

\section{Technique}

LDR brachytherapy is delivered using permanently implanted radioactive seeds that allow the delivery of very high doses of radiation to the target while limiting toxicities to organs at risk. Focal LDR brachytherapy would entail the insertion of such radioactive seeds into regions with well-identified lesions within the prostate while sparing the remainder of the gland and further reducing toxicity. Brachytherapy may offer certain advantages over other modalities, which include the geographic flexibility of shaping the treatment region by positioning the radioactive seeds as well as clear radiologic delineation of the region treated once implanted. As with whole-gland LDR brachytherapy, there may be

Table 3 Phase II hemigland protocol inclusion criteria

Men $\geq 21$ years of age with a life expectancy estimated to be $>10$ years Diagnosis of adenocarcinoma of the prostate confirmed by MSKCC or participating site pathology review

ECOG performance status ${ }^{85}$ of 0 or I

Prostate cancer clinical stage TIc-T2a

$\mathrm{PSA}<10 \mathrm{ng} / \mathrm{mL}$ (this will be the PSA level prompting the prostate biopsy) MRI evidence of one-sided disease performed within 3 months of registration

Prostate size $<60 \mathrm{~cm}^{3}$ at time of treatment; if the prostate is larger, hormonal therapy is allowed to achieve the required size

Abbreviations: ECOG, Eastern Cooperative Oncology Group; MRI, magnetic resonance imaging; MSKCC, Memorial Sloan-Kettering Cancer Center; PSA, prostate-specific antigen. 
Table 4 Phase II hemigland protocol exclusion criteria

Medically unfit for anesthesia

Evidence or suspicion of extracapsular extension on MRI

IPSS score $>18$

Unable to receive MRI

Prior radiotherapy for the current disease

Abbreviations: IPSS, International Prostate Symptom Score; MRI, magnetic resonance imaging.

differing opinions on certain aspects of delivering focal LDR brachytherapy. Methodological considerations include preplanning versus intraoperative planning, stranded versus loose seeds, and the choice of isotope. The focal brachytherapy consensus panel recommended a preplanned approach, although they did suggest that intraoperative planning software to monitor the procedure would be desirable..$^{57}$ In contrast, the Phase II hemigland brachytherapy protocol uses an intraoperative planning approach and utilizes information/ coordinates from the biopsy and the dominant tumor on the preoperative endorectal or pelvic coil MRI. In that protocol, the MRI information is fused to the intraoperative ultrasound images. ${ }^{8}$ With respect to stranded versus loose seeds, the consensus panel felt there may be a reduced potential for seed migration with stranded seeds, particularly at the periphery. However, some investigators have found that stranded seeds may migrate as well,${ }^{59}$ having potentially detrimental consequences in the setting of focal therapy. Loose seeds may offer the advantage of greater flexibility in geographic positioning of individual seeds, which may be of superior importance in focal treatment. This may be particularly true in cases of small lesions or lesions in close proximity to organs at risk. Isotopes used in LDR brachytherapy include $\mathrm{I}^{125}, \mathrm{Pd}^{103}$, and $\mathrm{Cs}^{131}$. Differences between the isotopes include relatively shorter half-lives with $\mathrm{Pd}^{103}$ and $\mathrm{Cs}^{131}$ when compared to $\mathrm{I}^{125}$, and higher energies with $\mathrm{I}^{125}$ and $\mathrm{Cs}^{131}$, when compared to $\mathrm{Pd}^{103}$. The guidelines for use of these isotopes should follow the general recommendations for whole-gland brachytherapy. For example, use of isotopes with shorter halflives, compared to edema half-life, could potentially lead to lower delivered dose than intended. Higher energy isotopes may be more forgiving in the context of geographic miss, due to a less acute dose fall off. However, care should be taken when placing seeds of higher energy in the proximity of sensitive structures, such as the urethra. The choice of methodology may best be chosen based on the institution's standard approach and familiarity with delivering wholegland brachytherapy.

With respect to planning volume definitions and dosimetric parameters, the Phase II hemigland brachytherapy protocol defines the gross target volume as the visible disease on preoperative MRI that can be fused to the intraoperative ultrasound images. The clinical target volume represents a $3 \mathrm{~mm}$ margin beyond the gross target volume and the planning target volume would represent the involved hemilobe of the prostate gland. Normal tissue constraints include urethra (volume, in percent) V100 $<130 \%$ of the prescription dose and a rectal $\mathrm{V} 100<100 \%$ of the prescription dose. In general, the computed tomography (CT) unit should be employed for the focal brachytherapy procedure, to confirm accurate placement of the seeds. Specific to the Phase II protocol, if it were determined based on the scan that there was a deficient dose region; this could be corrected with the placement of additional seeds after regeneration of the treatment plan and prior to the reversal of anesthesia. An example of a hemigland implant, with contours and isodose lines is shown in Figure 1. In comparison, a representative image of a focal implant, with contours and isodose lines, is shown in Figure 2.

Similar to whole-gland brachytherapy, routine postimplantation CT scan should be obtained for quality-assurance assessment of the adequacy of the implanted region. For whole-gland brachytherapy, the American Brachytherapy Society (ABS) recommends a postimplantation CT scan to be performed within 60 days of the implant. The optimum timing of the $\mathrm{CT}$ to minimize edema-derived dosimetry error is radionuclide specific: 16 days \pm 4 days for $\mathrm{Pd}^{103}$ and $\mathrm{Cs}^{131}$; and 30 days \pm 7 days for $\mathrm{I}^{125}$. A number of dosimetric parameters for the prostate, rectum, and urethra to assess implant quality have also been defined. ${ }^{60}$ These recommendations can be used as a guide to assess the adequacy of a partially implanted prostate. While the prostatic D90 (dose, in Gy and percent, received by $90 \%$ of the volume) for whole-gland brachytherapy has been used, due to the reduced target volume in partial-gland brachytherapy, its significance in relation to outcome is in question. However, the V100 and V150 for a partial-gland target, whether hemigland or a focal target, should be assessed. A V100 of at least $95 \%$ is desired while the V150 is radionuclide specific, but generally should be equal or less than $50 \%-60 \%$. For the urethra, the V150 (in volume), V5 (in percent), V30 (in percent), and the V100 for the rectum have been defined. ${ }^{61}$ Dosimetric goals for the urethra and rectum in whole-gland brachytherapy include a V5 $<150 \%$ and V30 $<125 \%$ for the urethra and a V100 of $<1 \mathrm{~cm}^{3}$ on day 1 and $<1.3 \mathrm{~cm}^{3}$ on day 30 dosimetry for the rectum. ${ }^{61}$ These goals can be considered acceptable constraints for partial-prostate brachytherapy dosimetry.

Other critical structures important for erectile dysfunction have been evaluated, including the internal pudendal 


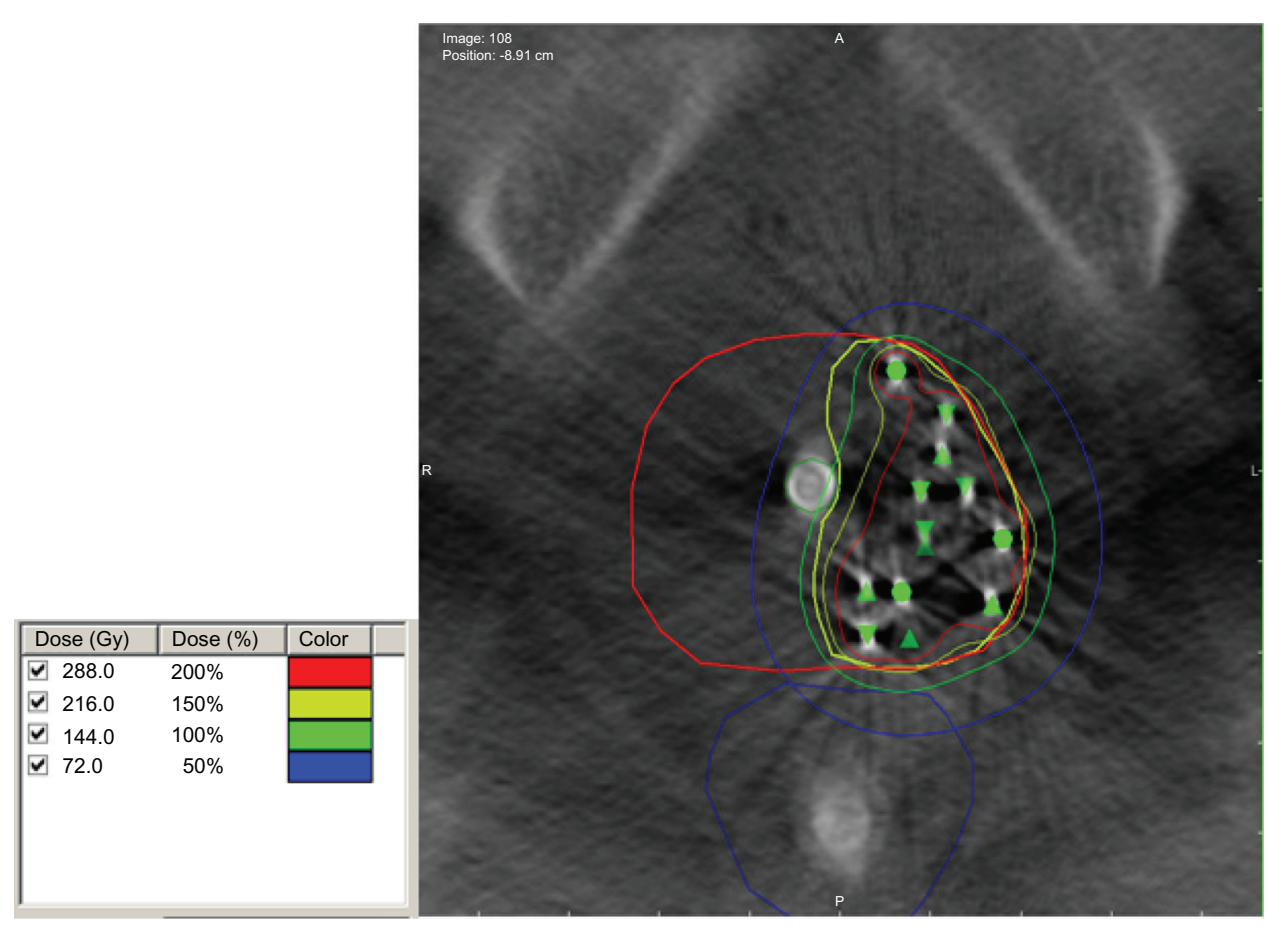

Figure I Hemigland brachytherapy implant.

Note: Computed tomography (contours of the prostate in red, target in yellow, rectum in blue. $100 \%, 150 \%$, and $200 \%$ isodose lines in green, yellow, and red). The green triangles and circles indicate seed positions.

artery, penile bulb, and neurovascular bundles; however, this is up for debate and is not recommended to be reported routinely. ${ }^{62-64}$

\section{Post-treatment care}

Patients should be evaluated on a regular basis post-treatment in a fashion similar to that performed for whole-gland therapy. In addition, the assessment of quality of life would be of particular importance, as a goal of focal therapy would be to reduce potential toxicities. Assessments would focus on erectile, urinary, and rectal function, as well as overall general health. A number of quality-of-life scales have been developed and used that could be applied to this population. The Memorial Sloan-Kettering Cancer Center Prostate-Health Related Quality of Life Questionnaire ${ }^{65}$ includes erectile function assessments derived from the Erectile Function Domain of

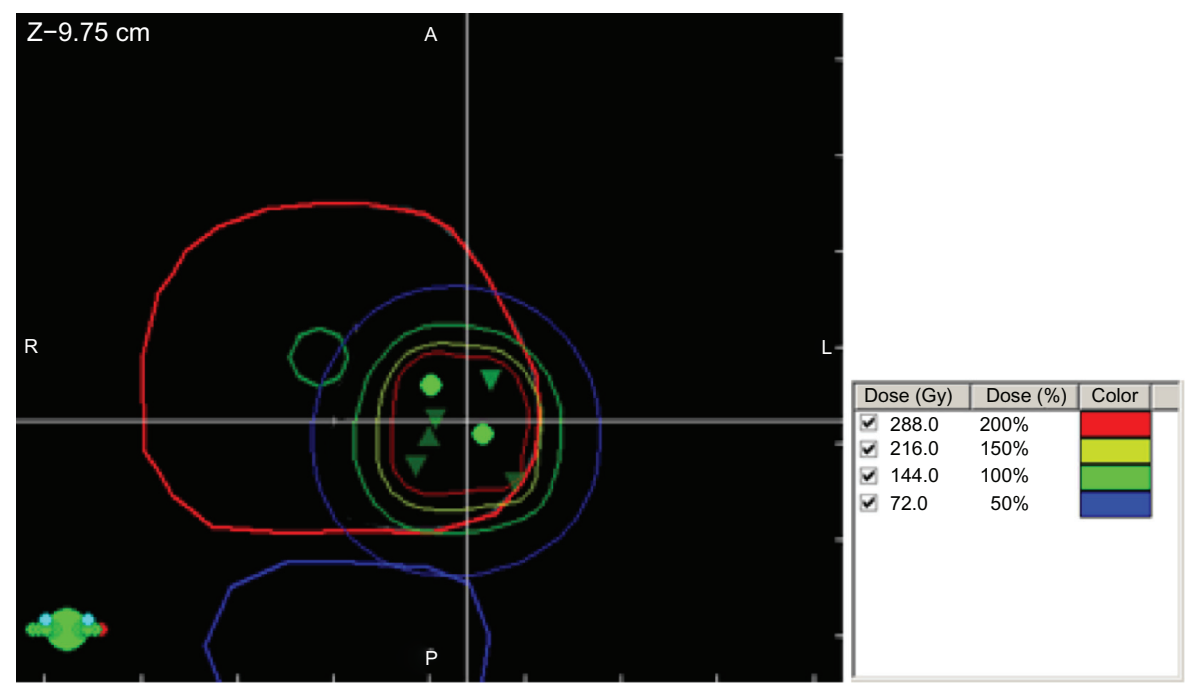

Figure 2 Representative image of a focal brachytherapy implant.

Notes: Contours of the prostate in red, urethra in green, and rectum in blue. The $100 \%, 150 \%$, and $200 \%$ isodose lines are in green, yellow, and red. The green triangles and circles indicate seed positions. 
the International Index of Erectile Function Questionnaire, ${ }^{66}$ as well as urinary and rectal function, and overall general health assessments. Other assessment scales include the IPSS, Expanded Prostate Cancer Index Composite, Short Form-36, International Index of Erectile Function 15, European Organisation for Research and Treatment of Cancer (EORTC) quality of life questionnaire C30, EORTC quality of life questionnaire $\operatorname{Pr} 25$, pain score, and urinary diaries. ${ }^{67-70}$

One challenge of patients treated with focal brachytherapy is finding the optimal method to monitor for disease recurrence. While some focal experiences to date have used the traditional method of PSA as a measure of outcome, the traditional definitions of PSA failure would not fully apply, due to the presence of normal prostatic tissue that was not treated. One proposal for better prediction of clinical failure following focal therapy is PSA velocity greater than $0.75 \mathrm{ng} / \mathrm{mL}$ per year, in addition to nadir $+2 .{ }^{9}$ Significant changes in PSA could be used as an indicator to warrant further tests to rule out recurrence, but it is clear that PSA evaluations alone would not be an effective method of assessing response or monitoring for recurrence. Other biomarkers to follow prostate cancer are being studied and could find utility in following patients with partial prostate treatment. These include transmembrane protease serine 2-ETS (erythroblast transformation-specific) related gene fusion, ${ }^{71}$ the phosphatase and tensin homolog gene, prostate cancer gene 3 , and urinary engrailed-2. ${ }^{72}$

An advantage with partial prostate treatment is that digital rectal examination could be helpful in detecting new abnormalities in the untreated region, which could warrant further investigations, such as a biopsy. However, similar to whole-gland treatment, a digital rectal examination in the treated area may be difficult to interpret due to soft tissue changes, such as fibrosis, post-treatment.

As previously discussed, multiparametric MRI, with functional sequences that include diffusion weighting, dynamic contrast enhancement, and MRI spectroscopy, is a major advancement in imaging that will play a vital role in identifying patients that will be appropriate for partial prostate brachytherapy. Postirradiation, there are morphologic changes that include inflammation, atrophy, and fibrosis that can pose challenges with traditional MRI evaluation. The addition of multiparametric MRI would be helpful to identify recurrent lesions in the treated area or new lesions in the untreated area. Its value has been demonstrated in detecting recurrent disease after HIFU ablation $^{73,74}$ or whole-gland external beam radiotherapy, ${ }^{75,76}$ and has been described in the setting of recurrence after focal therapy with HIFU, cryotherapy, external radiation, and brachytherapy. ${ }^{77}$

Interval biopsy, in conjunction with multiparametric MRI, should be considered as a follow-up tool. By sampling all areas of the prostate, potential recurrent lesions in the treated area or new lesions in the untreated areas can be identified. This is the only method to confirm the presence of disease. The follow-up regimen, outlined by the ongoing Phase II hemigland brachytherapy protocol, includes clinic evaluations at $3,6,12,18$, and 24 months after implantation, an MRI at 6 months and 24 months, and a transrectal or transperineal biopsy at 12 months and 24 months after the brachytherapy implant.

Failure after initial partial-prostate brachytherapy may occur either as a recurrence in the treated area, as a new lesion in the untreated areas, or both. In the setting of recurrence after whole-gland radiation, there are a number of recognized salvage treatment options, which include HIFU, cryotherapy, external radiation, radical prostatectomy, or brachytherapy. ${ }^{78-80}$ However, it should be noted that each modality, as a salvage, has its own toxicity profiles to consider, and there is no consensus as to the optimal option after whole-gland radiation. ${ }^{81}$ Because partial-gland brachytherapy is in its infancy, the optimal management of salvage for recurrent disease or retreatment of patients with new disease is even less clear, due to the limited experience. However, the salvage experiences for whole-gland therapy could be extrapolated as options. In addition, it could be hypothesized that initial partial-gland brachytherapy allows for the possibility of less toxicity when compared to salvage experiences after whole-gland radiation as well as the possibility to offer further partial-gland therapy to recurrent or new disease.

The limiting factor for retreatment with partial-gland brachytherapy would involve the normal tissue constraints, with the goals of maintaining the combined dosimetric parameters of the urethra, bladder, and rectum below the currently accepted constraints used for whole-gland brachytherapy. Therefore, salvage of a recurrent region or treatment of a new region with further partial-gland brachytherapy could potentially be done so long as the combined normal tissue constraints are met. However, while the presence of the brachytherapy seeds allows for the radiological delineation of the previously treated region, it may be a challenge to accurately combine the dosimetric plans, particularly in areas to be retreated or with new lesions that are in proximity to the previously treated regions. It is clear that further development of planning systems for this purpose 
and greater experience is needed to explore partial-gland brachytherapy as an option for retreatment or salvage.

\section{Conclusion}

The use of whole-gland LDR brachytherapy for localized prostate cancer has been well established. In comparison, the use of focal LDR brachytherapy is still in its early stages. Other modalities have shown promise in delivering focal treatment with comparable outcomes and reduced toxicity compared with whole-gland therapy. Extending these experiences to LDR brachytherapy is promising because of the well-established and successful experience with LDR brachytherapy for whole-gland treatment, and the added advantages of flexibility and the ease of transitioning a technique at an institution that may already have an established whole-gland brachytherapy program. The reduction in toxicity can be of significant importance particularly in a well-selected population. These include reduced rectal, urinary, and sexual toxicities. While it has not been established, it could also be hypothesized that an additional advantage of focal therapy could be the preservation of gland function and possibly the maintenance of fertility. A focal approach may also allow for an intermediary management option between active surveillance and initiating whole-gland therapy. ${ }^{82}$ With the advent of recent developments in ultrasound and MRI as well as advances and standardization of tissue sampling and interpretation, the appropriate population with unifocal, low-risk disease may be identified as optimal candidates to consider for focal therapy. Reduction in toxicity may lead to improved quality of life without the reduction in outcome. Post-treatment monitoring will have its challenges since the PSA alone would be an inadequate measure for disease recurrence. Digital rectal examination, multiparametric MRI, and interval biopsy will be important components of detecting disease recurrence in the treated area or new disease in the untreated areas. The optimal management for disease recurrence after partial-gland brachytherapy is unclear and the options may need to be considered on a case by case basis. While there is limited data for the use of partial-gland LDR brachytherapy, there are ongoing studies evaluating hemigland and focal approaches, and further analysis and studies are required to establish the validation and feasibility of offering focal LDR brachytherapy as an option to selected patients.

\section{Disclosure}

Yoshiya Yamada is a consultant for Varian Medical Systems, Palo Alto, CA, USA, and a member of the Speakers Bureau for the Institute for Medical Education. The authors report no other conflicts of interest in this work.

\section{References}

1. National Cancer Institute [homepage on the Internet]. Previous version SEER cancer statistics review, 1975-2009 (Vintage 2009 Populations). US National Institutes of Health; 2012 [updated August 20, 2012]. Available from http://seer.cancer.gov/csr/1975_2009_pops09/index. html. Accessed June 23, 2013.

2. Heidenreich A, Bellmunt J, Bolla M, et al. EAU guidelines on prostate cancer. Part 1: screening, diagnosis, and treatment of clinically localised disease. Eur Urol. 2011;59(1):61-71.

3. Sylvester JE, Grimm PD, Wong J, Galbreath RW, Merrick G, Blasko JC. Fifteen-year biochemical relapse-free survival, cause-specific survival, and overall survival following I(125) prostate brachytherapy in clinically localized prostate cancer: Seattle experience. Int J Radiat Oncol Biol Phys. 2011;81(2):376-381.

4. Stock RG, Cesaretti JA, Stone NN. Disease-specific survival following the brachytherapy management of prostate cancer. Int J Radiat Oncol Biol Phys. 2006;64(3):810-816.

5. Zelefsky MJ, Kuban DA, Levy LB, et al. Multi-institutional analysis of long-term outcome for stages T1-T2 prostate cancer treated with permanent seed implantation. Int J Radiat Oncol Biol Phys. 2007;67(2): 327-333.

6. Sanda MG, Dunn RL, Michalski J, et al. Quality of life and satisfaction with outcome among prostate-cancer survivors. $N$ Engl J Med. 2008;358(12):1250-1261.

7. Cosset JM, Wakil G, Pierrat N, Cathelineau X, Marchand V, Vallancien G. Poster 710. Focal brachytherapy for prostate cancer: a pilot study. Radiotherapy and Oncology. 2011;99:S283.

8. Memorial Sloan-Kettering Cancer Center. Phase II study assessing the potential for reduced toxicity using focal brachytherapy early stage, low volume in prostate cancer. In: ClinicalTrials.gov [website on the Internet]. New York, NY: Memorial Sloan-Kettering Cancer Center; 2011 [updated May 13, 2013]. Available from: http://clinicaltrials. gov/ct2/show/NCT01354951?term=focal+prostate\&rank=5. NLM identifier: NCT01354951. Accessed June 23, 2013.

9. Nguyen PL, Chen MH, Zhang Y, et al. Updated results of magnetic resonance imaging guided partial prostate brachytherapy for favorable risk prostate cancer: implications for focal therapy. J Urol. 2012;188(4): 1151-1156.

10. Kamrava M, Chung MP, Kayode O, et al. Focal high-dose-rate brachytherapy: a dosimetric comparison of hemigland vs conventional whole-gland treatment. Brachytherapy. Epub 2013 Feb 11.

11. Miralbell R, Mollà M, Rouzaud M, et al. Hypofractionated boost to the dominant tumor region with intensity modulated stereotactic radiotherapy for prostate cancer: a sequential dose escalation pilot study. Int J Radiat Oncol Biol Phys. 2010;78(1):50-57.

12. Aluwini S, van Rooij P, Hoogeman M, Kirkels W, Kolkman-Deurloo IK, Bangma C. Stereotactic body radiotherapy with a focal boost to the MRI-visible tumor as monotherapy for low- and intermediate-risk prostate cancer: early results. Radiat Oncol. 2013;8:84.

13. Schick U, Popowski Y, Nouet P, et al. High-dose-rate brachytherapy boost to the dominant intra-prostatic tumor region: hemi-irradiation of prostate cancer. Prostate. 2011;71(12):1309-1316.

14. Zaider M, Zelefsky MJ, Lee EK, et al. Treatment planning for prostate implants using magnetic-resonance spectroscopy imaging. Int $J$ Radiat Oncol Biol Phys. 2000;47(4):1085-1096.

15. Cooperberg MR, Lubeck DP, Meng MV, Mehta SS, Carroll PR. The changing face of low-risk prostate cancer: trends in clinical presentation and primary management. J Clin Oncol. 2004;22(11): 2141-2149.

16. Bott SR, Ahmed HU, Hindley RG, Abdul-Rahman A, Freeman A, Emberton $M$. The index lesion and focal therapy: an analysis of the pathological characteristics of prostate cancer. BJU Int. 2010;106(11): $1607-1611$. 
17. Karavitakis M, Winkler M, Abel P, Livni N, Beckley I, Ahmed HU. Histological characteristics of the index lesion in whole-mount radical prostatectomy specimens: implications for focal therapy. Prostate Cancer Prostatic Dis. 2011;14(1):46-52.

18. Muto S, Yoshii T, Saito K, Kamiyama Y, Ide H, Horie S. Focal therapy with high-intensity-focused ultrasound in the treatment of localized prostate cancer. Jpn J Clin Oncol. 2008;38(3):192-199.

19. El Fegoun AB, Barret E, Prapotnich D, et al. Focal therapy with highintensity focused ultrasound for prostate cancer in the elderly. A feasibility study with 10 years follow-up. Int Braz J Urol. 2011;37(2):213-219; discussion 220-222.

20. Bahn D, de Castro Abreu AL, Gill IS, et al. Focal cryotherapy for clinically unilateral, low-intermediate risk prostate cancer in 73 men with a median follow-up of 3.7 years. Eur Urol. 2012;62(1):55-63.

21. Lambert EH, Bolte K, Masson P, Katz AE. Focal cryosurgery: encouraging health outcomes for unifocal prostate cancer. Urology. 2007;69(6):1117-1120.

22. Onik G, Vaughan D, Lotenfoe R, Dineen M, Brady J. "Male lumpectomy": focal therapy for prostate cancer using cryoablation results in 48 patients with at least 2-year follow-up. Urol Oncol. 2008;26(5):500-505.

23. Keyes M, Miller S, Moravan V, et al. Predictive factors for acute and late urinary toxicity after permanent prostate brachytherapy: long-term outcome in 712 consecutive patients. Int J Radiat Oncol Biol Phys. 2009; 73(4):1023-1032.

24. Crook J, Fleshner N, Roberts C, Pond G. Long-term urinary sequelae following 125iodine prostate brachytherapy. J Urol. 2008;179(1): 141-145.

25. Stone NN, Stock RG. Long-term urinary, sexual, and rectal morbidity in patients treated with iodine-125 prostate brachytherapy followed up for a minimum of 5 years. Urology. 2007;69(2):338-342.

26. Keyes M, Miller S, Moravan V, et al. Urinary symptom flare in 712 125I prostate brachytherapy patients: long-term follow-up. Int J Radiat Oncol Biol Phys. 2009;75(3):649-655.

27. Keyes M, Spadinger I, Liu M, et al. Rectal toxicity and rectal dosimetry in low-dose-rate (125)I permanent prostate implants: a long-term study in 1006 patients. Brachytherapy. 2012;11(3):199-208.

28. Tran A, Wallner K, Merrick G, et al. Rectal fistulas after prostate brachytherapy. Int J Radiat Oncol Biol Phys. 2005;63(1):150-154.

29. Crook J, Borg J, Evans A, et al. 10-year experience with I-125 prostate brachytherapy at the Princess Margaret Hospital: results for 1,100 patients. Int J Radiat Oncol Biol Phys. 2011;80(5):1323-1329.

30. Pollack A, Zagars GK, Starkschall G, et al. Prostate cancer radiation dose response: results of the MD Anderson phase III randomized trial. Int J Radiat Oncol Biol Phys. 2002;53(5):1097-1105.

31. Halpern EJ, Frauscher F, Strup SE, Nazarian LN, O'Kane P, Gomella LG. Prostate: high-frequency Doppler US imaging for cancer detection. Radiology. 2002;225(1):71-77.

32. Mitterberger M, Horninger W, Pelzer A, et al. A prospective randomized trial comparing contrast-enhanced targeted versus systematic ultrasound guided biopsies: impact on prostate cancer detection. Prostate. 2007;67(14):1537-1542.

33. Newman JS, Bree RL, Rubin JM. Prostate cancer: diagnosis with color Doppler sonography with histologic correlation of each biopsy site. Radiology. 1995;195(1):86-90.

34. Roy C, Buy X, Lang H, Saussine C, Jacqmin D. Contrast enhanced color Doppler endorectal sonography of prostate: efficiency for detecting peripheral zone tumors and role for biopsy procedure. $J$ Urol. 2003;170(1):69-72.

35. Feleppa EJ, Fair WR, Liu T, et al. Three-dimensional ultrasound analyses of the prostate. Mol Urol. 2000;4(3):133-139.

36. Feleppa EJ, Fair WR, Tsai H, et al. Progress in two-dimensional and threedimensional ultrasonic tissue-type imaging of the prostate based on spectrum analysis and nonlinear classifiers. Mol Urol. 1999;3(3):303-310.

37. Braeckman J, Autier P, Soviany C, et al. The accuracy of transrectal ultrasonography supplemented with computer-aided ultrasonography for detecting small prostate cancers. BJU Int. 2008;102(11):1560-1565.
38. Hoyt K, Castaneda B, Zhang M, et al. Tissue elasticity properties as biomarkers for prostate cancer. Cancer Biomark. 2008;4(4-5): 213-225.

39. Nishida S, Kinoshita H, Mishima T, Kurokawa H, Sakaida N, Matsuda T. Prostate cancer detection by prebiopsy 3.0-Tesla magnetic resonance imaging. Int J Urol. 2011;18(9):653-658.

40. Puech P, Potiron E, Lemaitre L, et al. Dynamic contrast-enhancedmagnetic resonance imaging evaluation of intraprostatic prostate cancer: correlation with radical prostatectomy specimens. Urology. 2009;74(5): 1094-1099.

41. Villers A, Puech P, Mouton D, Leroy X, Ballereau C, Lemaitre L. Dynamic contrast enhanced, pelvic phased array magnetic resonance imaging of localized prostate cancer for predicting tumor volume: correlation with radical prostatectomy findings. $J$ Urol. 2006;176(6 Pt 1): 2432-2437.

42. Yamamura J, Salomon G, Buchert R, et al. MR imaging of prostate cancer: diffusion weighted imaging and (3D) hydrogen 1 (H) MR spectroscopy in comparison with histology. Radiol Res Pract. 2011; 2011:616852.

43. Arumainayagam N, Ahmed HU, Moore CM, Freeman A, Sohaib A, Kirkham A, et al. A negative multi-parametric MRI can rule out up to 97\% of clinically significant prostate cancer. Eur Urol Suppl. 2011;10(2): 66-67.

44. Kobus T, Hambrock T, Hulsbergen-van de Kaa CA, et al. In vivo assessment of prostate cancer aggressiveness using magnetic resonance spectroscopic imaging at $3 \mathrm{~T}$ with an endorectal coil. Eur Urol. 2011;60(5):1074-1080.

45. Dickinson L, Ahmed HU, Allen C, et al. Magnetic resonance imaging for the detection, localisation, and characterisation of prostate cancer: recommendations from a European consensus meeting. Eur Urol. 2011;59(4):477-494.

46. Bostwick DG, Meiers I. Prostate biopsy and optimization of cancer yield. Eur Urol. 2006;49(3):415-417.

47. Bostwick DG, Qian J, Drewnowska K, et al. Prostate needle biopsy quality in reduction by dutasteride of prostate cancer events study: worldwide comparison of improvement with investigator training and centralized laboratory processing. Urology. 2010;75(6): $1406-1410$

48. Onik G, Miessau M, Bostwick DG. Three-dimensional prostate mapping biopsy has a potentially significant impact on prostate cancer management. J Clin Oncol. 2009;27(26):4321-4326.

49. Falzarano SM, Zhou M, Hernandez AV, Moussa AS, Jones JS, Magi-Galluzzi C. Can saturation biopsy predict prostate cancer localization in radical prostatectomy specimens: a correlative study and implications for focal therapy. Urology. 2010;76(3):682-687.

50. Sinnott M, Falzarano SM, Hernandez AV, et al. Discrepancy in prostate cancer localization between biopsy and prostatectomy specimens in patients with unilateral positive biopsy: implications for focal therapy. Prostate. 2012;72(11):1179-1186.

51. Hambrock T, Somford DM, Hoeks C, et al. Magnetic resonance imaging guided prostate biopsy in men with repeat negative biopsies and increased prostate specific antigen. J Urol. 2010;183(2):520-527.

52. Ahmed HU, Hu Y, Carter T, et al. Characterizing clinically significant prostate cancer using template prostate mapping biopsy. $J$ Urol. 2011;186(2):458-464.

53. Horninger W, Berger AP, Rogatsch H, et al. Characteristics of prostate cancers detected at low PSA levels. Prostate. 2004;58(3):232-237.

54. Masterson TA, Cheng L, Mehan RM, Koch MO. Tumor focality does not predict biochemical recurrence after radical prostatectomy in men with clinically localized prostate cancer. $J$ Urol. 2011;186(2):506-510.

55. Ohori M, Eastham JA, Koh H, Kuroiwa K, Slawin KM, Wheeler TM, et al. Is focal therapy reasonable in patients with early stage prostate cancer $(\mathrm{CaP})$ - an analysis of radical prostatectomy (RP) specimens. J Urol. 2006;Suppl 175:507.

56. Arora R, Koch MO, Eble JN, Ulbright TM, Li L, Cheng L. Heterogeneity of Gleason grade in multifocal adenocarcinoma of the prostate. Cancer. 2004;100(11):2362-2366. 
57. Langley S, Ahmed HU, Al-Qaisieh B, et al. Report of a consensus meeting on focal low dose rate brachytherapy for prostate cancer. $B J U$ Int. 2012;109 Suppl 1:7-16.

58. Urology Specialists, PC. International Prostate Symptom Score (I-PSS). Middlebury, CT, USA: Urology Specialists, PC; 2013. Available from: http://www.urospec.com/uro/Forms/ipss.pdf. Accessed June 23, 2013.

59. Saibishkumar EP, Borg J, Yeung I, Cummins-Holder C, Landon A, Crook J. Sequential comparison of seed loss and prostate dosimetry of stranded seeds with loose seeds in 125I permanent implant for low-risk prostate cancer. Int J Radiat Oncol Biol Phys. 2009;73(1):61-68.

60. Davis BJ, Horwitz EM, Lee WR, et al; American Brachytherapy Society. American Brachytherapy Society consensus guidelines for transrectal ultrasound-guided permanent prostate brachytherapy. Brachytherapy. 2012;11(1):6-19.

61. Salembier C, Lavagnini P, Nickers P, et al; GEC ESTRO PROBATE Group. Tumour and target volumes in permanent prostate brachytherapy: a supplement to the ESTRO/EAU/EORTC recommendations on prostate brachytherapy. Radiother Oncol. 2007;83(1):3-10.

62. Buyyounouski MK, Horwitz EM, Uzzo RG, et al. The radiation doses to erectile tissues defined with magnetic resonance imaging after intensitymodulated radiation therapy or iodine-125 brachytherapy. Int J Radiat Oncol Biol Phys. 2004;59(5):1383-1391.

63. Gillan C, Kirilova A, Landon A, Yeung I, Pond G, Crook J. Radiation dose to the internal pudendal arteries from permanent-seed prostate brachytherapy as determined by time-of-flight MR angiography. Int $J$ Radiat Oncol Biol Phys. 2006;65(3):688-693.

64. Merrick GS, Butler WM, Wallner KE, et al. The importance of radiation doses to the penile bulb vs crura in the development of postbrachytherapy erectile dysfunction. Int J Radiat Oncol Biol Phys. 2002;54(4):1055-1062.

65. Befort CA, Zelefsky MJ, Scardino PT, Borrayo E, Giesler RB, Kattan MW. A measure of health-related quality of life among patients with localized prostate cancer: results from ongoing scale development. Clin Prostate Cancer. 2005;4(2):100-108.

66. Rosen RC, Riley A, Wagner G, Osterloh IH, Kirkpatrick J, Mishra A. The international index of erectile function (IIEF): a multidimensional scale for assessment of erectile dysfunction. Urology. 1997;49(6): 822-830.

67. Barry MJ, Fowler Jr. FJ, O’Leary MP, et al. The American Urological Association symptom index for benign prostatic hyperplasia. J Urol. 1992;148(5):1549-1557.

68. Wei JT, Dunn RL, Litwin MS, et al. Development and Validation of the Expanded Prostate Cancer Index Composite (EPIC) for Comprehensive Assessment of Health-Related Quality of Life in Men with Prostate Cancer. Urology. 2000;56(6):899-905.

69. Groenvold M, Klee MC, Sprangers MA, et al. Validation of the EORTC QLQ-C30 quality of life questionnaire through combined qualitative and quantitative assessment of patient-observer agreement. J Clin Epidemiol. 1997;50(4):441-450.

70. van Andel G, Bottomley A, Fosså SD, et al. An international field study of the EORTC QLQ-PR25: a questionnaire for assessing the healthrelated quality of life of patients with prostate cancer. Eur J Cancer. 2008;44(16):2418-2424
71. Rajput AB, Miller MA, De Luca A, et al. Frequency of the TMPRSS2: ERG gene fusion is increased in moderate to poorly differentiated prostate cancers. J Clin Pathol. 2007;60(11):1238-1243.

72. Morgan R, Boxall A, Bhatt A, et al. Engrailed-2 (EN2): a tumor specific urinary biomarker for the early diagnosis of prostate cancer. Clin Cancer Res. 2011;17(5):1090-1098.

73. Kim CK, Park BK, Lee HM, Kim SS, Kim E. MRI techniques for prediction of local tumor progression after high-intensity focused ultrasonic ablation of prostate cancer. AJR Am J Roentgenol. 2008;190(5): $1180-1186$

74. Rouvière O, Girouin N, Glas L, et al. Prostate cancer transrectal HIFU ablation: detection of local recurrences using T2-weighted and dynamic contrast-enhanced MRI. Eur Radiol. 2010;20(1):48-55.

75. Arumainayagam N, Kumaar S, Ahmed HU, et al. Accuracy of multiparametric magnetic resonance imaging in detecting recurrent prostate cancer after radiotherapy. BJU Int. 2010;106(7):991-997.

76. Haider MA, Chung P, Sweet J, et al. Dynamic contrast-enhanced magnetic resonance imaging for localization of recurrent prostate cancer after external beam radiotherapy. Int J Radiat Oncol Biol Phys. 2008;70(2):425-430.

77. De Visschere PJ, De Meerleer GO, Fütterer JJ, Villeirs GM. Role of MRI in follow-up after focal therapy for prostate carcinoma. AJR Am J Roentgenol. 2010;194(6):1427-1433.

78. Allen GW, Howard AR, Jarrard DF, Ritter MA. Management of prostate cancer recurrences after radiation therapy-brachytherapy as a salvage option. Cancer. 2007;110(7):1405-1416.

79. Nguyen PL, D'Amico AV, Lee AK, Suh WW. Patient selection, cancer control, and complications after salvage local therapy for postradiation prostate-specific antigen failure: a systematic review of the literature. Cancer. 2007;110(7):1417-1428.

80. Chalasani V, Martinez CH, Lim D, Chin J. Salvage HIFU for recurrent prostate cancer after radiotherapy. Prostate Cancer Prostatic Dis. 2009;12(2):124-129.

81. Boukaram C, Hannoun-Levi JM. Management of prostate cancer recurrence after definitive radiation therapy. Cancer Treat Rev. Apr 2010;36(2):91-100.

82. Tsivian M, Abern MR, Polascik TJ. Evolution of the concept of focal therapy for prostate cancer. Oncology (Williston Park). 2013;27(1): 64-68.

83. Ahmed HU, Hindley RG, Dickinson L, et al. Focal therapy for localised unifocal and multifocal prostate cancer: a prospective development study. Lancet Oncol. 2012;13(6):622-632.

84. Ahmed HU, Freeman A, Kirkham A, et al. Focal therapy for localized prostate cancer: a phase I/II trial. J Urol. 2011;185(4):1246-1254.

85. Eastern Cooperative Oncology Group [homepage on the Internet]. ECOG performance status; 2011 [updated July 27, 2006]. Available from: http://www.ecog.org/general/perf_stat.html. Accessed June 23, 2013.
Cancer Management and Research

\section{Publish your work in this journal}

Cancer Management and Research is an international, peer-reviewed open access journal focusing on cancer research and the optimal use of preventative and integrated treatment interventions to achieve improved outcomes, enhanced survival and quality of life for the cancer patient The journal welcomes original research, clinical \& epidemiological

\section{Dovepress}

studies, reviews \& evaluations, guidelines, expert opinion \& commentary, case reports \& extended reports. The manuscript management system is completely online and includes a very quick and fair peerreview system, which is all easy to use. Visit http://www.dovepress.com/ testimonials.php to read real quotes from published authors. 